\title{
Gigantomastia with mastitis during pregnancy in a patient with well controlled thyroid and lupus disease - a case report

\author{
J Prague ${ }^{1}$, L Geddes ${ }^{1}$, F Kaplan ${ }^{1}$
} \\ ${ }^{1}$ Department of Endocrinology - Lister Hospital, East and North Herts Trust, Hertfordshire
}

\section{Introduction}

- Gigantomastia is a rare condition characterised by excessive benign breast growth

- Thought to occur secondary to a change in the concentration of, or sensitivity to, circulating hormones (e.g. puberty/pregnancy)

- Isolated case reports in literature describe some medical conditions as triggers e.g systemic lupus

- Can cause significant physical and psychological symptoms

- Potential for conservative, medical and surgical management options

- 39-year-old female

\section{Case report}

- Normal BMI

- Conceived after in vitro fertilisation therapy

- Past history included:

- Graves' disease - well controlled on prophylthiouracil

- Systemic lupus erythematosus (SLE) - well controlled on hydroxychloroquine and aspirin

- bilateral breast fibroadenomas - resected 2010

- ectopic pregnancy - 2008

- Gradual increase in breast size correlating with her menstrual cycle starting a few months after her salpingectomy - brassiere cup increased from C to E at time of embryo transplantation

- Continued increase in breast size during her pregnancy (1 cup size/3 weeks)

- Started on empirical bromocriptine at 30 weeks

- Suffered with chronic breast and lower back pain

- Required inpatient admission for IV antibiotics at 33 weeks for urinary tract infection and mastitis

- At 34 weeks + 4 days ultrasound showed oligohydramnios thought secondary to the excessive breast tissue so proceeded to caesarean-section, which was uneventful

- As she chose not to breastfeed she continued on bromocriptine to suppress lactation

- After two further episodes of mastitis within 4 weeks of delivery and chronic pain she proceeded to reduction mammoplasty

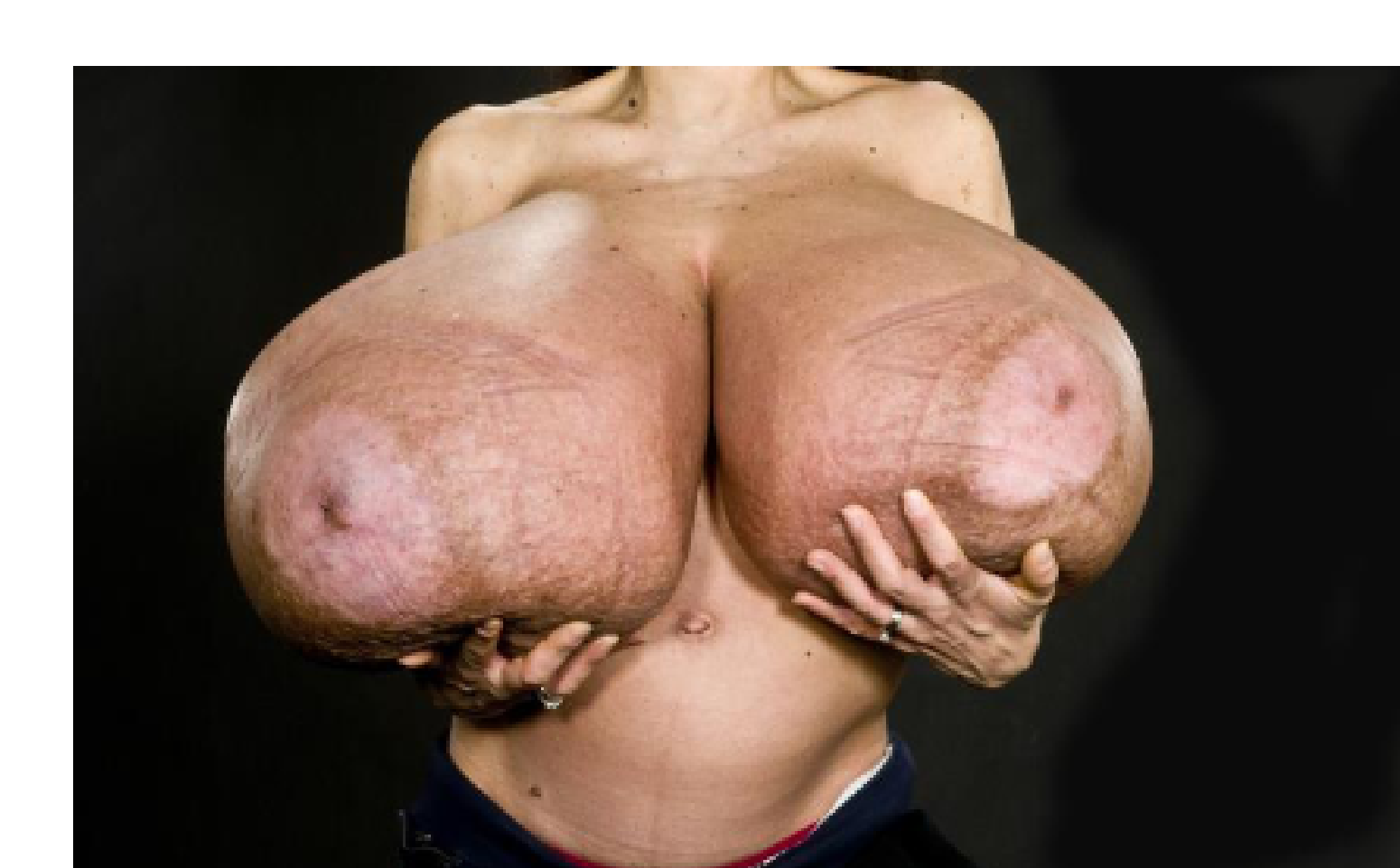

Anterior view

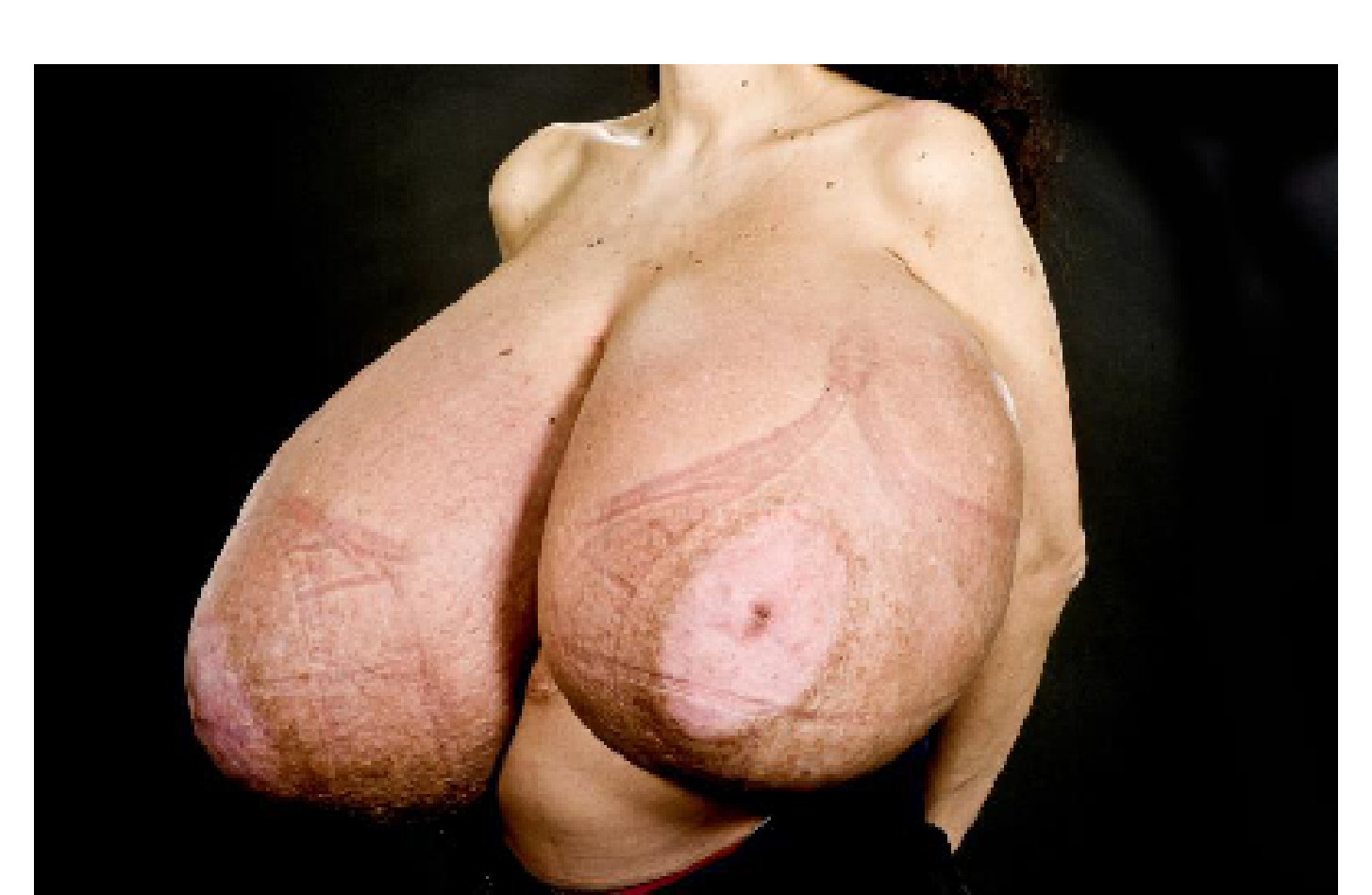

Left oblique

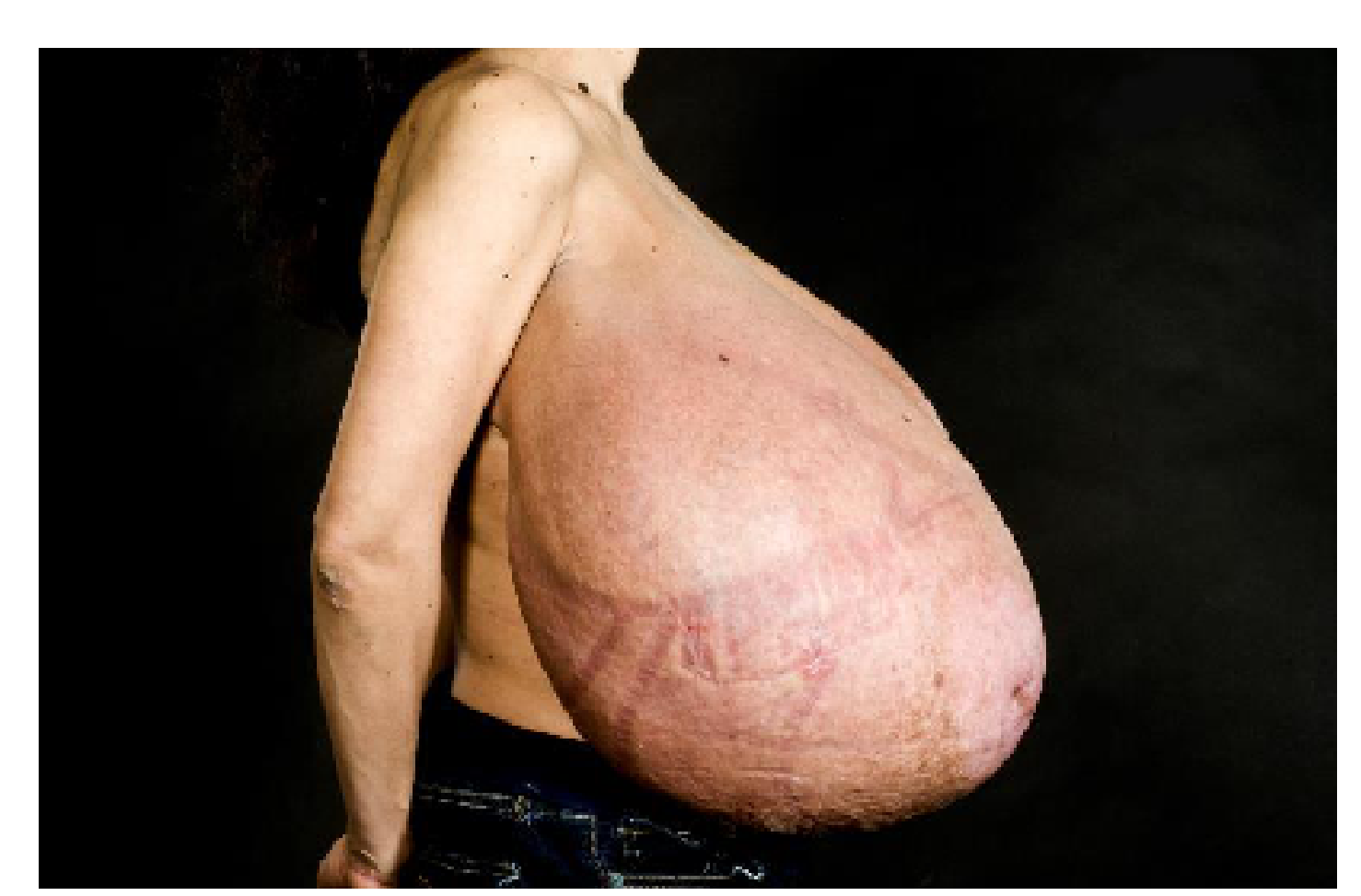

Right lateral

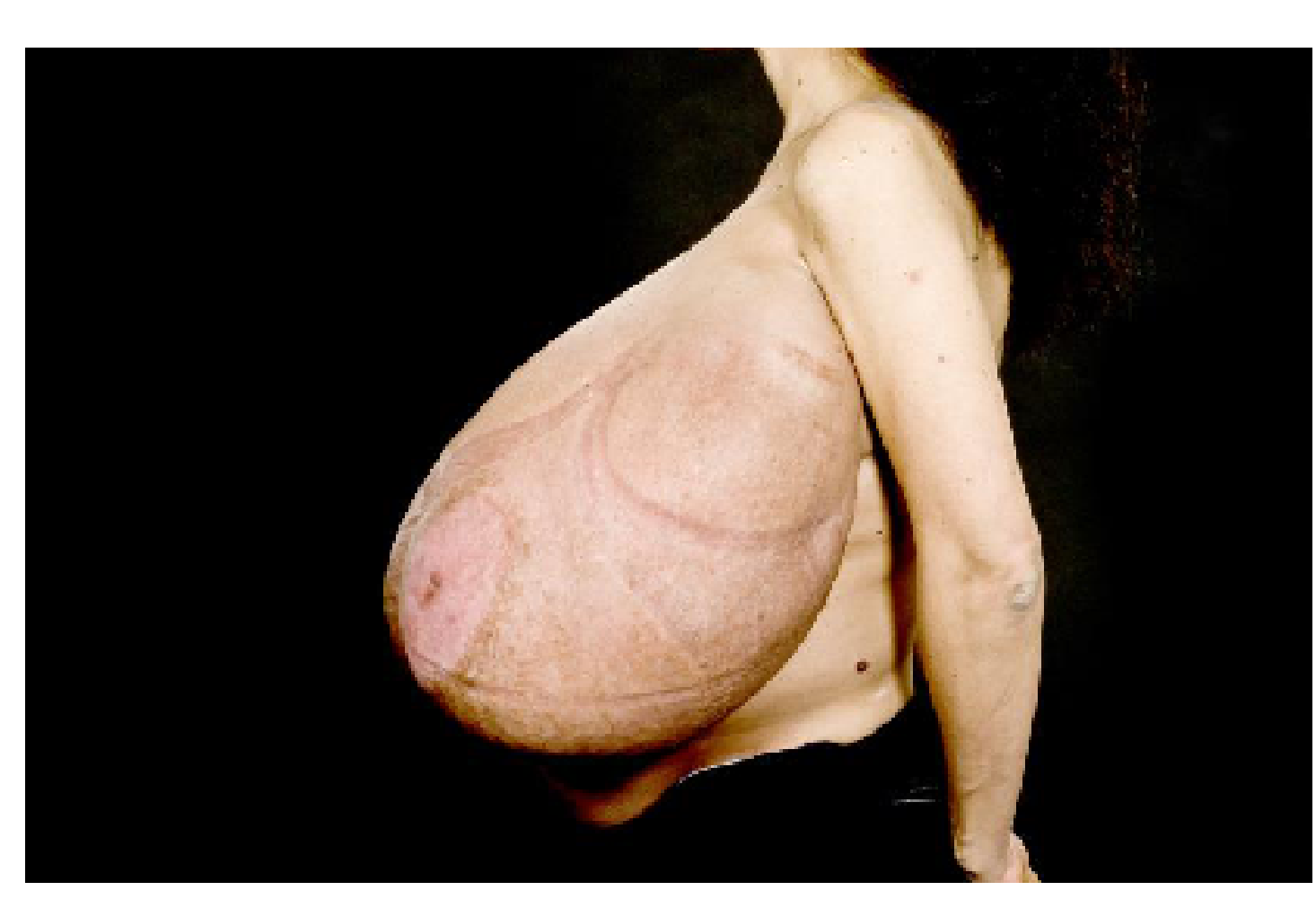

Left lateral

- Uncomplicated; total weight of tissue removed $6.53 \mathrm{~kg}$ ( $12 \%$ of her body weight)

- Histology confirmed benign proliferative change with no atypia

\section{Discussion}

- Gigantomastia is a rare, benign condition but is especially disabling to the pregnant woman and fetus

- Excellent outcomes can be achieved with surgery

- Isolated case reports in the literature suggest autoimmune medical conditions may be trigger factors

- To our knowledge this is the first documented case in a patient with autoimmune thyroid disease and SLE

References: Le EN et al, Lupus 2009; Towaine P et al, JCEM 2005; Dancey A et al, J Plastic, Reconstruct and Aesthetic Surgery 2006; Swelstad MR et al, Plastic Reconstr Surgery 2006

Corresponding Author: Julia Prague at juliaprague@nhs.net 